9. Kojima Y, Furubayashi K, Kawahata T, Mori H, Komano J. Circulation of distinct Treponema pallidum strains in individuals with heterosexual orientation and men who have sex with men. J Clin Microbiol. 2019;57:e01148-18. https://doi.org/10.1128/ JCM.01148-18

10. Staudová B, Strouhal M, Zobaníková M, Cejková D, Fulton LL, Chen L, et al. Whole genome sequence of the Treponema pallidum subsp. endemicum strain Bosnia A: the genome is related to yaws treponemes but contains few loci similar to syphilis treponemes. PLoS Negl Trop Dis. 2014;8:e3261. https://doi.org/10.1371/ journal.pntd.0003261

Address for correspondence: Takuya Kawahata, Osaka Institute of Public Health, 1-3-69 Nakamichi, Higashinari-ku, Osaka 537-0025, Japan; email:kawahata@iph.osaka.jp

\section{Multidrug-Resistant Klebsiella pneumoniae ST307 in Traveler Returning from Puerto Rico to Dominican Republic}

\section{Rita Rojas, Nenad Macesic, Gilda Tolari, Anel Guzman, Anne-Catrin Uhlemann}

\author{
Author affiliations: Hospital General Plaza de la Salud, Santo \\ Domingo, Dominican Republic (R. Rojas, G. Tolari, A. Guzman); \\ Monash University, Melbourne, Victoria, Australia (N. Macesic); \\ Columbia University Medical Center, New York, New York, USA \\ (N. Macesic, A.-C. Uhlemann)
}

DOI: https://doi.org/10.3201/eid2508.171730

We report $b / a_{\mathrm{KPC}-2}$-harboring carbapenem-resistant Klebsiella pneumoniae in an emerging sequence type 307 lineage in a traveler returning from Puerto Rico to the Dominican Republic. Phylogenetic analyses indicate regional dissemination of this highly drug-resistant clone across the Americas, underscoring the need for adequate surveillance and infection control efforts to prevent further spread.

$\mathrm{C}$ arbapenemase-resistant Enterobacteriaceae (CRE), in particular carbapenem-resistant Klebsiella pneumoniae (CRKp), represent a serious threat to public health (1). CRKp infections have been associated with high mortality rates, up to $50 \%$ in some studies (2). In resource- limited regions, such as the Dominican Republic, multiple challenges hinder efforts to contain CRE infections, including lack of novel antimicrobial drugs, inability to monitor drug levels of potentially toxic treatment regimens, and absence of molecular tools to investigate outbreaks and potential spread.

In fall 2015, a 66-year-old woman with diabetes mellitus, hepatitis $\mathrm{C}$ virus infection, and end-stage renal disease on hemodialysis was admitted to a hospital in the Dominican Republic for fever, anorexia, chills, and myalgia. On day 3, her blood culture tested positive for K. pneumoniae. She had been admitted to a hospital in Puerto Rico a few months before and had been treated for a multidrug-resistant bacterial infection.

The $K$. pneumoniae isolate from the patient was nonsusceptible to all tested antimicrobial drugs except polymyxins (Appendix Table 1, http://wwwnc.cdc.gov/EID/ article/25/8/17-1730-App1.pdf). We began combination therapy with a loading dose of colistin, then $100 \mathrm{mg}$ postdialysis, plus ertapenem (150 $\mathrm{mg}$ postdialysis) and fosfomycin ( $2 \mathrm{~g} 3 \times / \mathrm{d})$. We implemented infection control measures by placing the patient in a single room and using gloves, gowns, masks, and a dedicated stethoscope. Despite initial improvement, the patient died on day 25 after admission.

Whole-genome sequencing revealed that the patient isolate, NR6025, was of the emerging sequence type 307 (ST307) (3) and closely related ( $\leq 185$ SNPs) to several international ST307 isolates of similar phenotype (Figure). Of note, this isolate was most closely related, within 36 SNPs, to an isolate recovered from a patient in New York, NY, USA, who also had been hospitalized in Puerto Rico in 2016 (4). This finding raises the possibility that both patients acquired CRE in Puerto Rico and their infections subsequently developed in their home countries.

In silico resistance gene detection demonstrated that $b l a_{\mathrm{KPC}-2}$, on Tn4401e, was likely the mechanism of carbapenem resistance for this isolate. Moreover, the meropenem MIC was $>32 \mu \mathrm{g} / \mathrm{mL}$, consistent with high carbapenem MICs observed in the ST307 Tn4401e isolates (4) from New York, suggesting association with a strong promoter. In addition, the isolate harbored a large repertoire of acquired-resistance genes, including additional $\beta$-lactamase genes CTX-M-15, SHV-100, OXA-1, and TEM-1D (Appendix Table 1). The isolate contained IncFIBK, ColRNA1, and IncA/C2 plasmid replicons; IncA/C plasmid encodes for $b l a_{\mathrm{KPC}-2}, b l a_{\mathrm{TEM}}, s u l I, a a d B, a a c 6$, and qacE, which has been implicated in chlorhexidine resistance.

A case of CRKp was described from Medellin, Colombia, in 2005, and subsequent CRKp infections have been reported in Mexico, in South America in Brazil, Argentina, and Venezuela, and in the Caribbean in Cuba, Puerto Rico, and Trinidad and Tobago (5-7). In many of these studies, CRKp isolates were mainly accounted for 

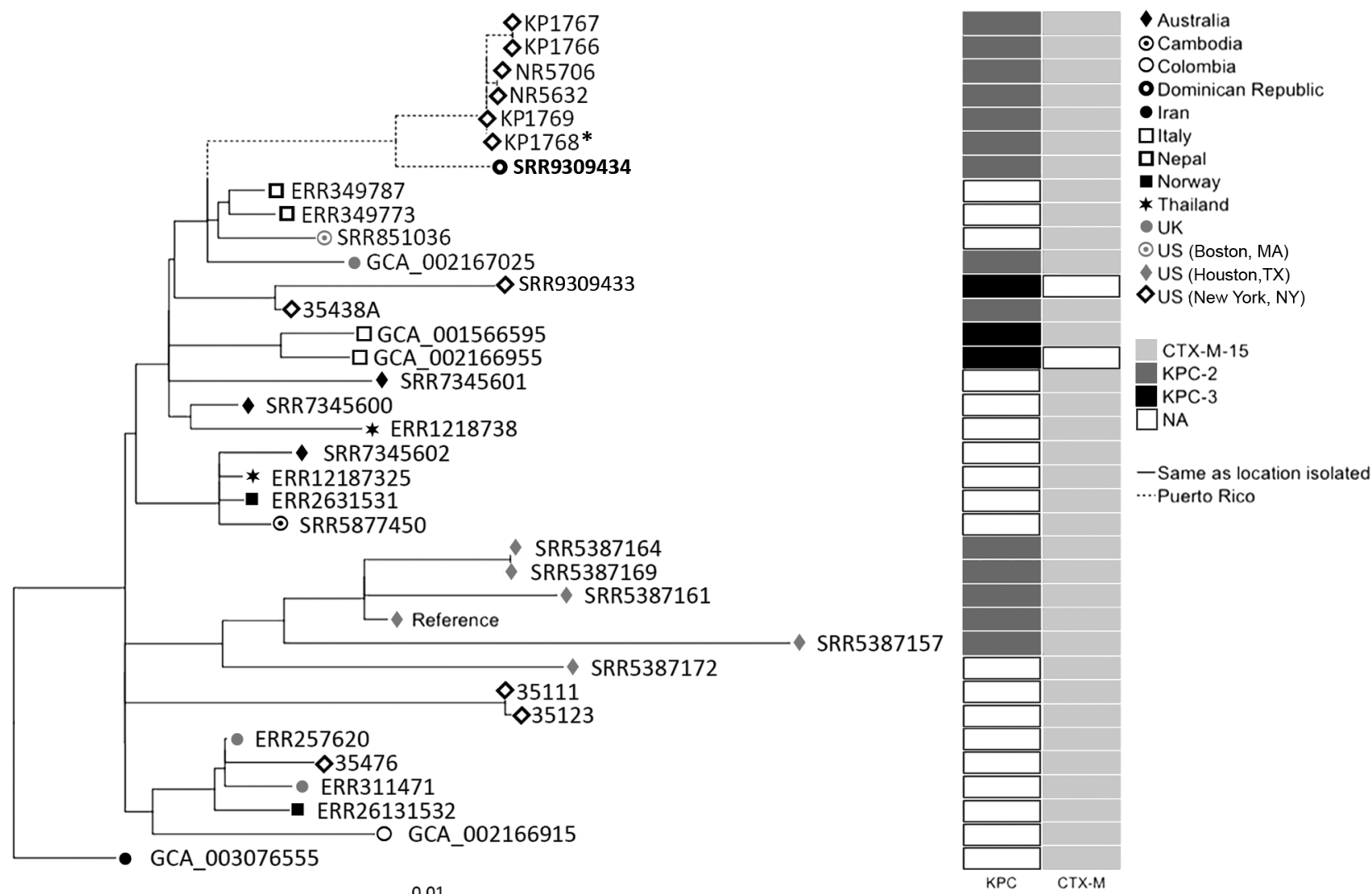

0.01

Figure. Maximum-likelihood phylogenetic tree of geographically diverse Klebsiella pneumoniae sequence type 307 isolates based on 860 concatenated single-nucleotide polymorphisms, extracted from an alignment length of 5,248,133 bp. Bold indicates isolate from a traveler from Puerto Rico to the Dominican Republic (this study). Asterisk $\left(^{*}\right)$ indicates an isolate recovered from a patient admitted to a hospital in Puerto Rico during the same year as the case-patient in this study (4). bla gene types (KPC, CTX-M) are indicated. Scale bar indicates nucleotide substitutions per site. NA, not applicable.

by ST258 and ST512. The SENTRY Antimicrobial Surveillance Program showed that lla $_{\mathrm{KPC}-2}$-harboring CRE accounted for most CRE infections in Latin America and that the incidence rate has been rising sharply (8). These organisms also are prevalent in Puerto Rico, where a 6-month, PCR-based, islandwide hospital surveillance study conducted in 2011 found that 333/2,805 (11.9\%) K. pneumoniae isolates harbored $b l a_{\mathrm{KPC}}(9)$. However, little is known about CRKp genotypes in Puerto Rico.

Our case highlights the many challenges for controlling CRE infections in resource-limited countries like the Dominican Republic and accentuates the potential for international spread of CRKp through travel, particularly between resource-limited regions. Rapid molecular diagnostic tests for CRKp are not widely available, which can delay treatment. Optimal treatment regimens for CRKp remain controversial, but combination therapy could reduce risk for death compared with monotherapy (10). Our facility lacked the resources needed to monitor colistin drug levels, a major concern in particular in patients with underlying renal dysfunction.
Risk factors for acquisition of CRE in resource-limited settings are not well defined, potentially delaying diagnosis and implementation of infection control strategies. In our case, recent travel, healthcare contact, and unspecified exposure to antimicrobial drugs might have played a role in the patient's CRE infection. We did not observe additional CRKp infections at our institution during a 6-month follow-up period after this case. However, we were unable to institute an active molecular surveillance program. We cannot rule out silent transmission and colonization of other hospitalized patients or contacts. Even though 2 cases have now been linked to travel to Puerto Rico, no molecular epidemiologic data are available from that island. Future studies should target active surveillance for CRKp in the Caribbean.

Of note, although ST258 has been the dominant genotype of the CRE epidemic globally, the ST307 clone could be expanding disproportionately in some locations. For example, in Houston, Texas, USA, ST307 now accounts for more K. pneumoniae infections than ST258 (3). Moreover, ST307 Tn4401e bla KPC-2 $_{2}$ isolates showed high carbapenem 
MICs. Taken together, our data suggest that ST307 is highly drug resistant and harbors an extended repertoire of antimicrobial resistance genes, which might have accelerated its recent emergence and wide dissemination.

This work was supported by grants from the National Institutes of Health, National Institute of Allergy and Infectious Diseases R01 AI116939 (A.-C.U.). The funders had no role in study design, data collection, interpretation, or the decision to submit the work for publication. A.-C.U. has received research funding from Merck, unrelated to the current study. All other authors declare no conflict of interest.

\section{About the Author}

Dr. Rojas is medical director of Centro Medico Luperon and the hospital epidemiologist at Hospital General de la Plaza de la Salud in Santo Domingo, Dominican Republic. Her research interests include healthcare-associated infections and detection and control of carbapenemase-producing Enterobacteriaceae.

\section{References}

1. US Centers for Disease Control and Prevention. Antibiotic resistance threats in the United States, 2013. 2013 Apr [cited 2017 Oct 10]. http://www.cdc.gov/drugresistance/threat-report-2013/index.html

2. Zarkotou O, Pournaras S, Tselioti P, Dragoumanos V, Pitiriga V, Ranellou K, et al. Predictors of mortality in patients with bloodstream infections caused by KPC-producing Klebsiella pneumoniae and impact of appropriate antimicrobial treatment. Clin Microbiol Infect. 2011;17:1798-803. https://doi.org/10.1111/j.1469-0691.2011.03514.x

3. Long SW, Olsen RJ, Eagar TN, Beres SB, Zhao P, Davis JJ, et al. Population genomic analysis of 1,777 extended-spectrum betalactamase-producing Klebsiella pneumoniae isolates, Houston, Texas: unexpected abundance of clonal group 307. MBio. 2017;8:e0489-17. https://doi.org/10.1128/mBio.00489-17

4. Giddins MJ, Macesic N, Annavajhala MK, Stump S, Khan S, McConville TH, et al. Successive emergence of ceftazidimeavibactam resistance through distinct genomic adaptations in bla $_{\mathrm{KPC}-2}$-harboring Klebsiella pneumoniae sequence type 307 isolates. Antimicrob Agents Chemother. 2018;62:e02101-17.

5. Escandón-Vargas K, Reyes S, Gutiérrez S, Villegas MV. The epidemiology of carbapenemases in Latin America and the Caribbean. Expert Rev Anti Infect Ther. 2017;15:277-97. https://doi.org/10.1080/14787210.2017.1268918

6. Pasteran FG, Otaegui L, Guerriero L, Radice G, Maggiora R, Rapoport M, et al. Klebsiella pneumoniae carbapenemase-2, Buenos Aires, Argentina. Emerg Infect Dis. 2008;14:1178-80. https://doi.org/10.3201/eid1407.070826

7. Falco A, Ramos Y, Franco E, Guzmán A, Takiff H. A cluster of KPC-2 and VIM-2-producing Klebsiella pneumoniae ST833 isolates from the pediatric service of a Venezuelan Hospital. BMC Infect Dis. 2016;16:595. https://doi.org/10.1186/s12879016-1927-y

8. Castanheira M, Costello AJ, Deshpande LM, Jones RN. Expansion of clonal complex $258 \mathrm{KPC}-2$-producing Klebsiella pneumoniae in Latin American hospitals: report of the SENTRY Antimicrobial Surveillance Program. Antimicrob Agents Chemother. 2012; 56:1668-9, author reply $1670-1$. https://doi.org/10.1128/ AAC.05942-11

9. Robledo IE, Aquino EE, Vázquez GJ. Detection of the KPC gene in Escherichia coli, Klebsiella pneumoniae, Pseudomonas aeruginosa, and Acinetobacter baumannii during a PCR-based nosocomial surveillance study in Puerto Rico. Antimicrob Agents Chemother. 2011;55:2968-70. https://doi.org/10.1128/ AAC.01633-10

10. Morrill HJ, Pogue JM, Kaye KS, LaPlante KL. Treatment options for carbapenem-resistant Enterobacteriaceae infections. Open Forum Infect Dis. 2015;2:ofv050. https://doi.org/10.1093/ofid/ ofv050

Address for correspondence: Anne-Catrin Uhlemann, Columbia University, Department of Medicine, Division of Infectious Diseases, 630 W 168th St, New York, NY 10032, USA; email: au2110@columbia.edu

\section{Stefano Veraldi, Luigi Esposito, Paolo Pontini, Fabrizio Vaira, Gianluca Nazzaro}

Author affiliation: Università degli Studi di Milano, Milan, Italy

DOI: https://doi.org/10.3201/eid2508.181063

Orf (ecthyma contagiosum) is an infection of the skin caused by a DNA virus belonging to the genus Parapoxvirus. We recently observed 7 cases of orf in Muslim men living in the metropolitan area of Milan, Italy, who acquired the infection after the Feast of Sacrifice.

$\mathrm{O}$ $\mathrm{f}$ (ecthyma contagiosum) is an infection of the skin caused by a DNA virus of the genus Parapoxvirus, family Poxviridae. Skin lesions (e.g., vesicles, blisters, pustules, erosions, ulcers, papules, nodules) occur at sites of inoculation of the virus 3-15 days after infection. Hands are usually affected (1). The differential diagnosis for orf includes milker's nodule, anthrax, tularemia, fish tank granuloma, cutaneous leishmaniasis, pyogenic granuloma, and keratoacanthoma (1). The disease spontaneously heals within 6 weeks, although pain, bacterial superinfections, and regional lymphadenitis are possible (1). Treatment is based on topical antiseptics (1).

Orf virus usually infects sheep and goats. Humans are infected by handling infected meat from these animals; orf is considered an occupational disease in shepherds, shearers, veterinarians, butchers, and cooks (1). 